\title{
Solving Multiple Integrals Using Maple
}

\author{
Chii-Huei Yu ${ }^{1, *}$, Bing-Huei Chen ${ }^{2}$ \\ ${ }^{1}$ Department of Management and Information, Nan Jeon University of Science and Technology, Tainan City, 73746, Taiwan \\ ${ }^{2}$ Department of Electrical Engineering, Nan Jeon University of Science and Technology, Tainan City, 73746, Taiwan \\ *Corresponding Author: chiihuei@mail.njtc.edu.tw
}

Copyright (C) 2014 Horizon Research Publishing All rights reserved.

\begin{abstract}
The multiple integral problem is closely related to probability theory and quantum field theory. This paper uses the mathematical software Maple for the auxiliary tool to study three types of multiple integrals. We can obtain the infinite series forms of these three types of multiple integrals by using differentiation with respect to a parameter, differentiation term by term, and integration term by term. In addition, we provide some examples to do calculation practically. The research methods adopted in this study involved finding solutions through manual calculations and verifying these solutions by using Maple. This type of research method not only allows the discovery of calculation errors, but also helps modify the original directions of thinking from manual and Maple calculations. For this reason, Maple provides insights and guidance regarding problem-solving methods.
\end{abstract}

Keywords Multiple Integrals, Infinite Series Forms, Differentiation with Respect to a Parameter, Differentiation Term by Term, Integration Term By Term, Maple

\section{Introduction}

As information technology advances, whether computers can become comparable with human brains to perform abstract tasks, such as abstract art similar to the paintings of Picasso and musical compositions similar to those of Mozart, is a natural question. Currently, this appears unattainable. In addition, whether computers can solve abstract and difficult mathematical problems and develop abstract mathematical theories such as those of mathematicians also appears unfeasible. Nevertheless, in seeking for alternatives, we can study what assistance mathematical software can provide. This study introduces how to conduct mathematical research using the mathematical software Maple. The main reasons of using Maple in this study are its simple instructions and ease of use, which enable beginners to learn the operating techniques in a short period. By employing the powerful computing capabilities of Maple, difficult problems can be easily solved. Even when Maple cannot determine the solution, problem-solving hints can be identified and inferred from the approximate values calculated and solutions to similar problems, as determined by Maple. For this reason, Maple can provide insights into scientific research. Inquiring through an online support system provided by Maple or browsing the Maple website (www.maplesoft.com) can facilitate further understanding of Maple and might provide unexpected insights. For the instructions and operations of Maple, [1-7] can be adopted as references.

The multiple integral problem is closely related with probability theory and quantum field theory, and can refer to [8-9]. For this reason, the evaluation and numerical calculation of multiple integrals is important. In this study, we evaluate the following three types of $n$-tuple integrals

$$
\begin{gathered}
\int_{0}^{1} \cdots \int_{0}^{1} \prod_{i=1}^{n}\left(\ln x_{i}\right)^{m_{i}} \cdot \prod_{i=1}^{n} x_{i}^{\beta_{i}} \cdot \cos \left(\prod_{i=1}^{n} x_{i}^{\lambda_{i}}\right) d x_{1} \cdots d x_{n} \\
\int_{0}^{1} \cdots \int_{0}^{1} \prod_{i=1}^{n}\left(\ln x_{i}\right)^{m_{i}} \cdot \prod_{i=1}^{n} x_{i}^{\beta_{i}} \cdot \sin \left(\prod_{i=1}^{n} x_{i}^{\lambda_{i}}\right) d x_{1} \cdots d x_{n} \\
\int_{0}^{1} \cdots \int_{0}^{1} \prod_{i=1}^{n}\left(\ln x_{i}\right)^{m_{i}} \cdot \prod_{i=1}^{n} x_{i}^{\beta_{i}} \cdot \exp \left(\prod_{i=1}^{n} x_{i}^{\lambda_{i}}\right) d x_{1} \cdots d x_{n}
\end{gathered}
$$

where $n$ is a positive integer, $m_{i}$ are non-negative integers, and $\beta_{i}, \lambda_{i}$ are real numbers for all $i=1, . ., n$. We can obtain the infinite series forms of these three types of multiple integrals by using differentiation with respect to a parameter, differentiation term by term, and integration term by term ; these are the major results of this study (i.e., Theorems 1-3). In addition, we obtain three corollaries from the three theorems. For the study of related multiple integral problems can refer to [10-25]. On the other hand, we propose some multiple integrals to do calculation practically. The research methods adopted in this study involved finding solutions through manual calculations and verifying these solutions by using Maple. This type of research method not only allows the discovery of calculation errors, but also helps modify the original directions of thinking from manual and Maple calculations. Therefore, Maple provides insights and guidance regarding problem-solving methods. The following is the flowchart of the research method used in this paper. 


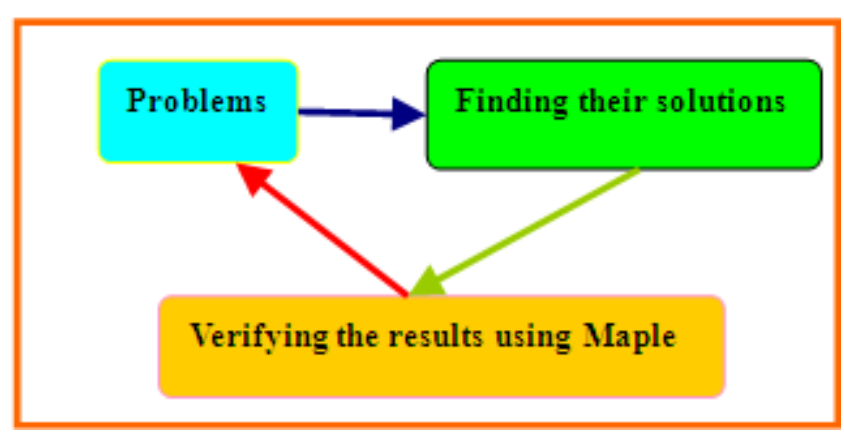

Figure 1. The flowchart of the research method used in this paper.

\section{Main Results}

Firstly, we introduce some notations and formulas used in this paper.

\subsection{Notations}

i. $\quad \prod_{i=1}^{n} c_{i}=c_{1} \times c_{2} \times \cdots \times c_{n}$, where $n$ is a positive integer, $c_{i}$ are real numbers for all $i=1, . ., n$.

ii. $\quad p !=p \times(p-1) \times \cdots \times 1$, where $p$ is a positive integer, and $0 !=1$.

\subsection{Formulas}

(A) $\cos u=\sum_{k=0}^{\infty} \frac{(-1)^{k}}{(2 k) !} u^{2 k}$, where $u$ is a real number.

(B) $\sin u=\sum_{k=0}^{\infty} \frac{(-1)^{k}}{(2 k+1) !} u^{2 k+1}$, where $u$ is a real number.

(C) $e^{u}=\sum_{k=0}^{\infty} \frac{1}{k !} u^{k}$, where $u$ is a real number.

Next, we introduce three important theorems used in this study.

\subsection{Differentiation with Respect to a Parameter ([26]).}

Suppose $I=$ $\left[c_{1}, c_{2}\right] \times\left[a_{11}, a_{12}\right] \times\left[a_{21}, a_{22}\right] \times \cdots \times\left[a_{n 1}, a_{n 2}\right]$, and the $(n+1)$-variables function $f\left(\rho, x_{1}, x_{2}, \cdots, x_{n}\right)$ is defined on $I$. If $f\left(\rho, x_{1}, x_{2}, \cdots, x_{n}\right)$ and its partial derivative $\frac{\partial f}{\partial \rho}\left(\rho, x_{1}, x_{2}, \cdots, x_{n}\right)$ are continuous functions on $I$. Then $F(\rho)=\int_{a_{11}}^{a_{12}} \int_{a_{21}}^{a_{22}} \cdots \int_{a_{n 1}}^{a_{n 2}} f\left(\rho, x_{1}, x_{2}, x_{3}, \cdots, x_{n}\right) d x_{1} d x_{2} \cdots d x_{n}$ is differentiable on the open interval $\left(c_{1}, c_{2}\right)$, and its derivative

$\frac{d}{d \rho} F(\rho)=\int_{a_{11}}^{a_{12}} \int_{a_{21}}^{a_{22}} \cdots \int_{a_{n 1}}^{a_{n 2}} \frac{\partial f}{\partial \rho}\left(\rho, x_{1}, x_{2}, x_{3}, \cdots, x_{n}\right) d x_{2} d x_{3} \cdots d x_{n}$ for all $\rho \in\left(c_{1}, c_{2}\right)$.

\subsection{Differentiation Term by Term ([27, p230])}

For all non-negative integers $k$, if the functions $g_{k}:(a, b) \rightarrow R$ satisfy the following three conditions : (i) there exists a point $x_{0} \in(a, b)$ such that $\sum_{k=0}^{\infty} g_{k}\left(x_{0}\right)$ is convergent, (ii) all functions $g_{k}(x)$ are differentiable on open interval $(a, b)$, (iii) $\sum_{k=0}^{\infty} \frac{d}{d x} g_{k}(x)$ is uniformly convergent on $(a, b)$. Then $\sum_{k=0}^{\infty} g_{k}(x)$ is uniformly convergent and differentiable on $(a, b)$, and its derivative

$$
\frac{d}{d x} \sum_{k=0}^{\infty} g_{k}(x)=\sum_{k=0}^{\infty} \frac{d}{d x} g_{k}(x) .
$$

\subsection{Integration Term by Term ([27, p269]).}

Suppose $\left\{g_{n}\right\}_{n=0}^{\infty}$ is a sequence of Lebesgue integrable functions defined on an inteval $I$. If $\sum_{n=0}^{\infty} \int_{I}\left|g_{n}\right|$ is convergent, then $\int_{I} \sum_{n=0}^{\infty} g_{n}=\sum_{n=0}^{\infty} \int_{I} g_{n}$.

The following is the first major result in this study, we determine the infinite series form of the multiple integral (1).

2.6. Theorem 1 Suppose $n$ is a positive integer, $m_{i}$ are non-negative integers, and $\beta_{i}, \lambda_{i}$ are real numbers, $\lambda_{i} \geq 0, \beta_{i}>-1$ for all $i=1, . ., n$. Then the $n$-tuple integral

$$
\begin{aligned}
& \int_{0}^{1} \cdots \int_{0}^{1} \prod_{i=1}^{n}\left(\ln x_{i}\right)^{m_{i}} \cdot \prod_{i=1}^{n} x_{i}^{\beta_{i}} \cdot \cos \left(\prod_{i=1}^{n} x_{i}^{\lambda_{i}}\right) d x_{1} \cdots d x_{n} \\
= & (-1)^{i=1} \sum_{i=1}^{n} m_{i} m_{i} ! \cdot \sum_{k=0}^{\infty} \frac{(-1)^{k}}{(2 k) ! \cdot \prod_{i=1}^{n}\left(2 \lambda_{i} k+\beta_{i}+1\right)^{m_{i}+1}}
\end{aligned}
$$

Proof Because

$$
\begin{gathered}
\prod_{i=1}^{n} x_{i}^{\beta_{i}} \cdot \cos \left(\prod_{i=1}^{n} x_{i}^{\lambda_{i}}\right) \\
=\prod_{i=1}^{n} x_{i} \beta_{i} \cdot \sum_{k=0}^{\infty} \frac{(-1)^{k}}{(2 k) !}\left(\prod_{i=1}^{n} x_{i}^{\lambda_{i}}\right)^{2 k}
\end{gathered}
$$

(By Formula (A)) 


$$
=\sum_{k=0}^{\infty} \frac{(-1)^{k}}{(2 k) !} \prod_{i=1}^{n} x_{i}^{2 \lambda_{i} k+\beta_{i}}
$$

Therefore,

$$
\begin{aligned}
& \int_{0}^{1} \cdots \int_{0}^{1} \prod_{i=1}^{n} x_{i} \beta_{i} \cdot \cos \left(\prod_{i=1}^{n} x_{i} \lambda_{i}\right) d x_{1} \cdots d x_{n} \\
= & \int_{0}^{1} \cdots \int_{0}^{1} \sum_{k=0}^{\infty} \frac{(-1)^{k}}{(2 k) !} \prod_{i=1}^{n} x_{i}^{2 \lambda_{i} k+\beta_{i}} d x_{1} \cdots d x_{n} \\
= & \sum_{k=0}^{\infty} \frac{(-1)^{k}}{(2 k) !} \int_{0}^{1} \cdots \int_{0}^{1} \prod_{i=1}^{n} x_{i}{ }^{2 \lambda_{i} k+\beta_{i}} d x_{1} \cdots d x_{n}
\end{aligned}
$$

( By integration term by term )

$$
\begin{aligned}
=\sum_{k=0}^{\infty} & \frac{(-1)^{k}}{(2 k) !} \cdot \prod_{i=1}^{n} \int_{0}^{1} x_{i}{ }^{2 \lambda_{i} k+\beta_{i}} d x_{i} \\
= & \sum_{k=0}^{\infty} \frac{(-1)^{k}}{(2 k) ! \cdot \prod_{i=1}^{n}\left(2 \lambda_{i} k+\beta_{i}+1\right)}
\end{aligned}
$$

Using differentiation with respect to a parameter and differentiation term by term, we differentiate $\beta_{i}$ by $m_{i}$ times on both sides of (6) for all $i=1, \cdots, n$. We obtain

$$
\begin{aligned}
& \int_{0}^{1} \cdots \int_{0}^{1} \prod_{i=1}^{n}\left(\ln x_{i}\right)^{m_{i}} \cdot \prod_{i=1}^{n} x_{i} \beta_{i} \cdot \cos \left(\prod_{i=1}^{n} x_{i}^{\lambda_{i}}\right) d x_{1} \cdots d x_{n} \\
& =(-1)^{i=1} m_{i=1}^{n} m_{i} ! \cdot \sum_{k=0}^{\infty} \frac{(-1)^{k}}{(2 k) ! \cdot \prod_{i=1}^{n}\left(2 \lambda_{i} k+\beta_{i}+1\right)^{m_{i}+1}}
\end{aligned}
$$

In Theorem 1 , taking $x_{i}=e^{-y_{i}}$ for all $i=1, . ., n$, we immediately have the following result.

2.7. Corollary 1 If the assumptions are the same as Theorem 1, then the $n$-tuple improper integral

$$
\begin{array}{r}
\int_{0}^{\infty} \cdots \int_{0}^{\infty} \prod_{i=1}^{n} y_{i}{ }^{m_{i}} \cdot \exp \left(-\sum_{i=1}^{n}\left(\beta_{i}+1\right) y_{i}\right) \cos \left(\exp \left(-\sum_{i=1}^{n} \lambda_{i} y_{i}\right)\right) d y_{1} \cdots d y_{n} \\
=\prod_{i=1}^{n} m_{i} ! \cdot \sum_{k=0}^{\infty} \frac{(-1)^{k}}{(2 k) ! \cdot \prod_{i=1}^{n}\left(2 \lambda_{i} k+\beta_{i}+1\right)^{m_{i}+1}}
\end{array}
$$

Next, we determine the infinite series forms of the multiple integral (2).

2.8. Theorem 2 Suppose $n$ is a positive integer, $m_{i}$ are non-negative integers, and $\beta_{i}, \lambda_{i}$ are real numbers, $\lambda_{i} \geq 0, \lambda_{i}+\beta_{i}>-1$ for all $i=1, . ., n$. Then the $n$ -tuple integral

$$
\int_{0}^{1} \cdots \int_{0}^{1} \prod_{i=1}^{n}\left(\ln x_{i}\right)^{m_{i}} \cdot \prod_{i=1}^{n} x_{i}^{\beta_{i}} \cdot \sin \left(\prod_{i=1}^{n} x_{i}^{\lambda_{i}}\right) d x_{1} \cdots d x_{n}
$$

$$
=(-1)^{\sum_{i=1}^{n} m_{i}} \prod_{i=1}^{n} m_{i} ! \cdot \sum_{k=0}^{\infty} \frac{(-1)^{k}}{(2 k+1) ! \cdot \prod_{i=1}^{n}\left(2 \lambda_{i} k+\lambda_{i}+\beta_{i}+1\right)^{m_{i}+1}}
$$

Proof Because

$$
\begin{gathered}
\prod_{i=1}^{n} x_{i}^{\beta_{i}} \cdot \sin \left(\prod_{i=1}^{n} x_{i}^{\lambda_{i}}\right) \\
=\prod_{i=1}^{n} x_{i} \beta_{i} \cdot \sum_{k=0}^{\infty} \frac{(-1)^{k}}{(2 k+1) !}\left(\prod_{i=1}^{n} x_{i}^{\lambda_{i}}\right)^{2 k+1}
\end{gathered}
$$

(By Formula (B))

$$
=\sum_{k=0}^{\infty} \frac{(-1)^{k}}{(2 k+1) !} \prod_{i=1}^{n} x_{i}^{2 \lambda_{i} k+\lambda_{i}+\beta_{i}}
$$

Thus,

$$
\begin{gathered}
\int_{0}^{1} \cdots \int_{0}^{1} \prod_{i=1}^{n} x_{i}^{\beta_{i}} \cdot \sin \left(\prod_{i=1}^{n} x_{i}^{\lambda_{i}}\right) d x_{1} \cdots d x_{n} \\
=\int_{0}^{1} \cdots \int_{0}^{1} \sum_{k=0}^{\infty} \frac{(-1)^{k}}{(2 k+1) !} \prod_{i=1}^{n} x_{i}^{2 \lambda_{i} k+\lambda_{i}+\beta_{i}} d x_{1} \cdots d x_{n}
\end{gathered}
$$

(By (9))

$$
=\sum_{k=0}^{\infty} \frac{(-1)^{k}}{(2 k+1) !} \int_{0}^{1} \cdots \int_{0}^{1} \prod_{i=1}^{n} x_{i}^{2 \lambda_{i} k+\lambda_{i}+\beta_{i}} d x_{1} \cdots d x_{n}
$$

(Using integration term by term )

$$
\begin{array}{r}
=\sum_{k=0}^{\infty} \frac{(-1)^{k}}{(2 k+1) !} \cdot \prod_{i=1}^{n} \int_{0}^{1} x_{i}^{2 \lambda_{i} k+\lambda_{i}+\beta_{i}} d x_{i} \\
=\sum_{k=0}^{\infty} \frac{(-1)^{k}}{(2 k+1) ! \cdot \prod_{i=1}^{n}\left(2 \lambda_{i} k+\lambda_{i}+\beta_{i}+1\right)}
\end{array}
$$

Also, by differentiation with respect to a parameter and differentiation term by term, differentiating $\beta_{i}$ by $m_{i}$ times on both sides of (10) for all $i=1, \cdots, n$. We obtain

$$
\begin{aligned}
& \int_{0}^{1} \cdots \int_{0}^{1} \prod_{i=1}^{n}\left(\ln x_{i}\right)^{m_{i}} \cdot \prod_{i=1}^{n} x_{i}^{\beta_{i}} \cdot \sin \left(\prod_{i=1}^{n} x_{i} \lambda_{i}\right) d x_{1} \cdots d x_{n} \\
& =(-1)^{i=1} \prod_{i=1}^{n} m_{i} ! \cdot \sum_{k=0}^{\infty} \frac{(-1)^{k}}{(2 k+1) ! \cdot \prod_{i=1}^{n}\left(2 \lambda_{i} k+\lambda_{i}+\beta_{i}+1\right)^{m_{i}+1}}
\end{aligned}
$$

In Theorem 2, let $x_{i}=e^{-y_{i}}$ for $i=1, . ., n$, then the following result holds.

2.9. Corollary 2 If the assumptions are the same as Theorem 2, then the $n$-tuple improper integral 


$$
\begin{gathered}
\int_{0}^{\infty} \cdots \int_{0}^{\infty} \prod_{i=1}^{n} y_{i} m_{i} \cdot \exp \left(-\sum_{i=1}^{n}\left(\beta_{i}+1\right) y_{i}\right) \sin \left(\exp \left(-\sum_{i=1}^{n} \lambda_{i} y_{i}\right)\right) d y_{1} \cdots d y_{n} \\
=\prod_{i=1}^{n} m_{i} ! \cdot \sum_{k=0}^{\infty} \frac{(-1)^{k}}{(2 k+1) ! \prod_{i=1}^{n}\left(2 \lambda_{i} k+\lambda_{i}+\beta_{i}+1\right)^{m_{i}+1}}
\end{gathered}
$$

Finally, we obtain the infinite series forms of the multiple integral (3).

2.10. Theorem 3 If the assumptions are the same as Theorem 1 . Then the $n$-tuple integral

$$
\begin{aligned}
\int_{0}^{1} \cdots \int_{0}^{1} \prod_{i=1}^{n}\left(\ln x_{i}\right)^{m_{i}} \cdot \prod_{i=1}^{n} x_{i}^{\beta_{i}} \cdot \exp \left(\prod_{i=1}^{n} x_{i}^{\lambda_{i}}\right) d x_{1} \cdots d x_{n} \\
\quad=(-1)^{\sum_{i=1}^{n} m_{i}} \prod_{i=1}^{n} m_{i} ! \cdot \sum_{k=0}^{\infty} \frac{1}{k ! \cdot \prod_{i=1}^{n}\left(\lambda_{i} k+\beta_{i}+1\right)^{m_{i}+1}}
\end{aligned}
$$

Proof Because

$$
\begin{aligned}
& \prod_{i=1}^{n} x_{i}^{\beta_{i}} \cdot \exp \left(\prod_{i=1}^{n} x_{i}^{\lambda_{i}}\right) \\
= & \prod_{i=1}^{n} x_{i} \beta_{i} \cdot \sum_{k=0}^{\infty} \frac{1}{k !}\left(\prod_{i=1}^{n} x_{i}^{\lambda_{i}}\right)^{k}
\end{aligned}
$$

(Using Formula (C))

$$
=\sum_{k=0}^{\infty} \frac{1}{k !} \prod_{i=1}^{n} x_{i} \lambda_{i} k+\beta_{i}
$$

Hence,

$$
\begin{array}{r}
\int_{0}^{1} \cdots \int_{0}^{1} \prod_{i=1}^{n} x_{i}^{\beta_{i}} \cdot \exp \left(\prod_{i=1}^{n} x_{i}^{\lambda_{i}}\right) d x_{1} \cdots d x_{n} \\
=\int_{0}^{1} \cdots \int_{0}^{1} \sum_{k=0}^{\infty} \frac{1}{k !} \prod_{i=1}^{n} x_{i} \lambda_{i} k+\beta_{i} d x_{1} \cdots d x_{n} \quad \text { By } \\
=\sum_{k=0}^{\infty} \frac{1}{k !} \int_{0}^{1} \cdots \int_{0}^{1} \prod_{i=1}^{n} x_{i}^{\lambda_{i} k+\beta_{i}} d x_{1} \cdots d x_{n}
\end{array}
$$

( By integration term by term )

$$
\begin{array}{r}
=\sum_{k=0}^{\infty} \frac{1}{k !} \cdot \prod_{i=1}^{n} \int_{0}^{1} x_{i} \lambda_{i} k+\beta_{i} d x_{i} \\
=\sum_{k=0}^{\infty} \frac{1}{k ! \prod_{i=1}^{n}\left(\lambda_{i} k+\beta_{i}+1\right)}
\end{array}
$$

By differentiation with respect to a parameter and differentiation term by term, differentiating $\beta_{i}$ by $m_{i}$ times on both sides of (14) for all $i=1, \cdots, n$. We have

$$
\begin{aligned}
& \int_{0}^{1} \cdots \int_{0}^{1} \prod_{i=1}^{n}\left(\ln x_{i}\right)^{m_{i}} \cdot \prod_{i=1}^{n} x_{i}^{\beta_{i}} \cdot \exp \left(\prod_{i=1}^{n} x_{i}^{\lambda_{i}}\right) d x_{1} \cdots d x_{n} \\
& =(-1)^{i=1} \prod_{i=1}^{n} m_{i} ! \cdot \sum_{k=0}^{\infty} \frac{1}{k ! \cdot \prod_{i=1}^{n}\left(\lambda_{i} k+\beta_{i}+1\right)^{m_{i}+1}}
\end{aligned}
$$

In Theorem 3, let $x_{i}=e^{-y_{i}}$ for $i=1, . ., n$, the following result holds.

2.11. Corollary 3 If the assumptions are the same as Theorem 1, then the $n$-tuple improper integral

$$
\begin{aligned}
\int_{0}^{\infty} \cdots \int_{0}^{\infty} \prod_{i=1}^{n} y_{i} m_{i} \cdot \exp \left(-\sum_{i=1}^{n}\left(\beta_{i}+1\right) y_{i}\right) \exp \left(\exp \left(-\sum_{i=1}^{n} \lambda_{i} y_{i}\right)\right) d y_{1} \cdots d y_{n} \\
=\prod_{i=1}^{n} m_{i} ! \cdot \sum_{k=0}^{\infty} \frac{1}{k ! \cdot \prod_{i=1}^{n}\left(\lambda_{i} k+\beta_{i}+1\right)^{m_{i}+1}}
\end{aligned}
$$

\section{Examples}

In the following, for the three types of multiple integrals in this study, we provide some examples and use Theorems 1-3 and Corollaries 1-3 to determine the infinite series forms of these multiple integrals. On the other hand, we employ Maple to calculate the approximations of these multiple integrals and their solutions for verifying our answers.

3.1. Example 1 By Theorem 1, we obtain the double integral

$$
\begin{aligned}
& \int_{0}^{1} \int_{0}^{1}\left(\ln x_{1}\right)^{2}\left(\ln x_{2}\right)^{3} x_{1}{ }^{7 / 3} x_{2}{ }^{9 / 2} \cos \left(x_{1}{ }^{2 / 5} x_{2}{ }^{11 / 6}\right) d x_{1} d x_{2} \\
& =-12 \cdot \sum_{k=0}^{\infty} \frac{(-1)^{k}}{(2 k) ! \cdot(4 k / 5+10 / 3)^{3}(11 k / 3+11 / 2)^{4}}
\end{aligned}
$$

Next, we use Maple to verify the correctness of (16). $>\operatorname{evalf}\left(\right.$ Doubleint $\left((\ln (\mathrm{x} 1))^{\wedge} 2^{*}(\ln (\mathrm{x} 2))^{\wedge} 3^{*} \mathrm{x} 1^{\wedge}(7 / 3)^{*} \mathrm{x} 2^{\wedge}(9 / 2)\right.$ $\left.\left.* \cos \left(\mathrm{x} 1^{\wedge}(2 / 5) * \mathrm{x} 2^{\wedge}(11 / 6)\right), \mathrm{x} 1=0 . .1, \mathrm{x} 2=0 . .1\right), 14\right)$;

$$
-0.00034219267849328
$$

$>\operatorname{evalf}\left(-12 * \operatorname{sum}\left((-1)^{\wedge} \mathrm{k} /\left((2 * \mathrm{k}) ! *(4 * \mathrm{k} / 5+10 / 3)^{\wedge} 3 *(11 * \mathrm{k} / 3+\right.\right.\right.$

$\left.11 / 2)^{\wedge} 4\right), \mathrm{k}=0$..infinity), 14$)$;

$$
-0.00034219267849327
$$

3.2. Example 2 By Corollary 1, the triple improper integral $\int_{0}^{\infty} \int_{0}^{\infty} \int_{0}^{\infty} y_{1}^{3} y_{2} y_{3}{ }^{2} \exp \left(-2 y_{1}-3 y_{2}-4 y_{3}\right) \cos \left(\exp \left(-4 y_{1}-y_{2}-2 y_{3}\right)\right) d y_{1} d y_{2} d y_{3}$

$$
=1440 \cdot \sum_{k=0}^{\infty} \frac{(-1)^{k}}{(2 k) ! \cdot(8 k+2)^{4}(2 k+3)^{6}(4 k+4)^{3}}
$$

$>\operatorname{evalf(Tripleint}\left(\mathrm{y} 1 \wedge 3 * \mathrm{y} 2 \wedge 5^{*} \mathrm{y} 3 \wedge 2 \exp (-2 * \mathrm{y} 1-3 * \mathrm{y} 2-4 * \mathrm{y} 3) *\right.$ $\cos (\exp (-4 * \mathrm{y} 1-\mathrm{y} 2-2 * \mathrm{y} 3)), \mathrm{y} 1=0$..infinity, $\mathrm{y} 2=0$. infinity, $\mathrm{y} 3=0$ 
..infinity),14);

\subsection{4}

$>\operatorname{evalf}\left(1440 * \operatorname{sum}\left((-1)^{\wedge} \mathrm{k} /\left((2 * \mathrm{k}) !^{*}(8 * \mathrm{k}+2)^{\wedge} 4 *(2 * \mathrm{k}+3)^{\wedge} 6 *(4\right.\right.\right.$

$\left.* \mathrm{k}+4)^{\wedge} 3\right), \mathrm{k}=0$..infinity), 14$)$;

$$
0.0019290033484884
$$

3.3. Example 3 Using Theorem 2, we can evaluate the double integral

$$
\begin{gathered}
\int_{0}^{1} \int_{0}^{1}\left(\ln x_{1}\right)^{5}\left(\ln x_{2}\right)^{3} x_{1}{ }^{1 / 7} x_{2}{ }^{9 / 5} \sin \left(x_{1}{ }^{6 / 7} x_{2}{ }^{4 / 5}\right) d x_{1} d x_{2} \\
\quad=720 \cdot \sum_{k=0}^{\infty} \frac{(-1)^{k}}{(2 k+1) ! \cdot(12 k / 7+24 / 7)^{6}(8 k / 5+18 / 5)^{4}}
\end{gathered}
$$

$>$ evalf(Doubleint $\left((\ln (\mathrm{x} 1))^{\wedge} 5^{*}(\ln (\mathrm{x} 2))^{\wedge} 3^{*} \mathrm{x} 1^{\wedge}(11 / 7) * \mathrm{x} 2^{\wedge}(9 / 5\right.$

)$\left.\left.^{*} \sin \left(\mathrm{x} 1^{\wedge}(6 / 7) * \mathrm{x} 2^{\wedge}(4 / 5)\right), \mathrm{x} 1=0 . .1, \mathrm{x} 2=0 . .1\right), 14\right)$;

$$
0.0026301802197270
$$

$>\operatorname{evalf}\left(720 * \operatorname{sum}\left((-1)^{\wedge} \mathrm{k} /\left((2 * \mathrm{k}+1) ! *(12 * \mathrm{k} / 7+24 / 7)^{\wedge} 6 *(8 * \mathrm{k} / 5\right.\right.\right.$ $\left.+18 / 5)^{\wedge} 4\right), \mathrm{k}=0$..infinity), 14$)$;

$$
0.0026301802197270
$$

3.4. Example 4 By Corollary 2, the double improper integral

$$
\begin{gathered}
\int_{0}^{\infty} \int_{0}^{\infty} y_{1}{ }^{4} y_{2}{ }^{2} \exp \left(-2 y_{1}-7 y_{2}\right) \sin \left(\exp \left(-5 y_{1}-3 y_{2}\right)\right) d y_{1} d y_{2} \\
\quad=48 \cdot \sum_{k=0}^{\infty} \frac{(-1)^{k}}{(2 k+1) ! \cdot(10 k+7)^{5}(6 k+10)^{3}}
\end{gathered}
$$

$>\operatorname{evalf}($ Doubleint $(\mathrm{y} 1 \wedge 4 * \mathrm{y} 2 \wedge 2 * \exp (-2 * \mathrm{y} 1-7 * \mathrm{y} 2) * \sin (\exp (-5 *$ y1-3*y2)),y1=0..infinity,y2 $=0$..infinity),14);

$$
0.0000028545799098894
$$

$>\operatorname{evalf}\left(48 * \operatorname{sum}\left((-1)^{\wedge} \mathrm{k} /\left((2 * \mathrm{k}+1) ! *(10 * \mathrm{k}+7)^{\wedge} 5 *(6 * \mathrm{k}+10)^{\wedge} 3\right)\right.\right.$, $\mathrm{k}=0$..infinity), 14$)$;

\subsection{3}

3.5. Example 5 Using Theorem 3, the double integral

$$
\begin{aligned}
& \int_{0}^{1} \int_{0}^{1}\left(\ln x_{1}\right)^{2}\left(\ln x_{2}\right)^{7} x_{1}{ }^{1 / 4} x_{2}{ }^{3 / 8} \exp \left(x_{1}{ }^{4 / 9} x_{2}{ }^{5 / 11}\right) d x_{1} d x_{2} \\
& =-10080 \cdot \sum_{k=0}^{\infty} \frac{1}{k ! \cdot(4 k / 9+5 / 4)^{3}(5 k / 11+11 / 8)^{8}}
\end{aligned}
$$

$>\operatorname{evalf}\left(\right.$ Doubleint $\left((\ln (\mathrm{x} 1))^{\wedge} 2^{*}(\ln (\mathrm{x} 2))^{\wedge} 7^{*} \mathrm{x} 1^{\wedge}(1 / 4)^{*} \mathrm{x} 2^{\wedge}(3 / 8)\right.$

$\left.\left.* \exp \left(\mathrm{x} 1^{\wedge}(4 / 9) * \mathrm{x} 2^{\wedge}(5 / 11)\right), \mathrm{x} 1=0 . .1, \mathrm{x} 2=0 . .1\right), 14\right)$;

$$
-421.16580400909
$$

$>$ evalf(-10080* $\operatorname{sum}\left(1 /\left(\mathrm{k} ! *(4 * \mathrm{k} / 9+5 / 4)^{\wedge} 3 *(5 * \mathrm{k} / 11+11 / 8)^{\wedge} 8\right)\right.$ $, \mathrm{k}=0$..infinity), 14$)$;

\section{$-421.16580400908$}

3.6. Example 6 By Corollary 3, we obtain the double improper integral

$$
\begin{gathered}
\int_{0}^{\infty} \int_{0}^{\infty} y_{1} y_{2}{ }^{4} \exp \left(-2 y_{1}-3 y_{2}\right) \exp \left(\exp \left(-4 y_{1}-2 y_{2}\right)\right) d y_{1} d y_{2} \\
=24 \cdot \sum_{k=0}^{\infty} \frac{1}{k ! \cdot(4 k+2)^{2}(2 k+3)^{5}}
\end{gathered}
$$

$>\operatorname{evalf}($ Doubleint $(\mathrm{y} 1 * \mathrm{y} 2 \wedge 4 * \exp (-2 * \mathrm{y} 1-3 * \mathrm{y} 2) * \exp (\exp (-4 * \mathrm{y}$ $1-2 * \mathrm{y} 2)), \mathrm{y} 1=0$. infinity, $\mathrm{y} 2=0$. .infinity), 14$)$;

$$
0.024912197200303
$$

$>\operatorname{evalf}\left(24 * \operatorname{sum}\left(1 /\left(\mathrm{k} ! *(4 * \mathrm{k}+2)^{\wedge} 2 *(2 * \mathrm{k}+3)^{\wedge} 5\right), \mathrm{k}=0\right.\right.$..infinity $)$, $14)$;

$$
0.024912197200303
$$

\section{Conclusions}

As mentioned, evaluating the multiple integrals is important in probability theory and quantum field theory. In this study, we provide a new technique to solve three types of multiple integrals, and we hope this method can be applied in mathematical statistics or quantum physics. Simultaneously, the differentiation with respect to a parameter, the differentiation term by term and the integration term by term play significant roles in the theoretical inferences of this study. In fact, the application of the three theorems is extensive, and can be used to easily solve many difficult problems; we endeavor to conduct further studies on related applications.

\section{REFERENCES}

[1] F. Garvan, The Maple Book, London: Chapman \& Hall/CRC, 2001.

[2] C. T. J. Dodson and E. A. Gonzalez, Experiments in Mathematics Using Maple, New York: Springer-Verlag, 1995.

[3] R. J. Stroeker and J. F. Kaashoek, Discovering Mathematics with Maple : An Interactive Exploration for Mathematicians, Engineers and Econometricians, Basel: Birkhauser Verlag, 1999.

[4] D. Richards, Advanced Mathematical Methods with Maple, New York: Cambridge University Press, 2002.

[5] J. S. Robertson, Engineering Mathematics with Maple, New York: McGraw-Hill, 1996.

[6] C. Tocci and S. G. Adams, Applied Maple for Engineers and Scientists, Boston: Artech House, 1996.

[7] M. L. Abell and J. P. Braselton, Maple by Example, 3rd ed., New York: Elsevier Academic Press, 2005.

[8] F. Streit, On multiple integral geometric integrals and their applications to probability theory, Canadian Journal of Mathematics, Vol. 22, pp. 151-163, 1970.

[9] L. H. Ryder, Quantum Field Theory, 2nd ed., New York: Cambridge University Press, 1996.

[10] C. -H. Yu, Using Maple to study the multiple improper integral problem, Proceedings of IIE Asian Conference 2013, Taiwan, Vol. 1, pp. 625-632, 2013.

[11] C. $-\mathrm{H} . \mathrm{Yu}, \mathrm{A}$ study on the multiple improper integral 
problems, Journal of Hsin Sheng, Vol. 12, pp.175-194, 2013.

[12] C. - H. Yu, Application of Maple: taking the evaluation of double improper integrals as an example, Proceedings of the 7th International Conference on Information Technology, Taiwan, No.28, 2013.

[13] C. -H. Yu, Application of Maple: two types of improper integrals as examples, Proceedings of 2013 Information Technology and Management Symposium, Taiwan, B9-B14, 2013.

[14] C. -H. Yu, Application of Maple on solving some type of multiple integrals, Proceedings of the 8th Information Management and Practice Conference, Taiwan, TP20120128 2012.

[15] C. -H. Yu, Application of Maple: multiple integrals as examples, Proceedings of the 6th Cross-Strait Technology and Humanities Education and University-Industry Cooperation Seminar, Taiwan, EMD001, 2012.

[16] C. -H. Yu, Application of Maple on evaluating multiple improper integrals, Proceedings of the 6th IEEE/International Conference on Advanced Infocomm Technology, Taiwan, No. 00282, 2013.

[17] C. -H. Yu, Application of Maple on evaluating the double improper integrals, Proceedings of the Innovative Education and Learning Technology, 2013.

[18] C. -H. Yu, Using Maple to study multiple improper integrals, International Journal of Research in Information Technology, Vol. 1, Issue. 8, pp. 10-14, 2013.
[19] C. -H. Yu, A study on two types of multiple integrals, Computational Research, Vol. 2, No. 1, pp. 1-4, 2014.

[20] C. -H. Yu, A study on multiple improper integrals using Maple, International Journal of Physics and Mathematical Sciences, Vol. 3, No. 3, pp. 55-60, 2013.

[21] C. -H. Yu, The evaluation of two types of multiple improper integrals, Proceedings of 2012 Chang Yun Ka Alliance of Colleges Symposium, Taiwan, M-7, 2012.

[22] C.-H. Yu, A study on the multiple improper integral problems with Maple, Applied Mechanics and Materials, Vols. 479-480 (2014), pp 828-833, 2013.

[23] C. -H. Yu, Application of Maple: the evaluation of double integral as an example, Proceedings of 2013 International Symposium on Intercultural Communication, Taiwan, pp. 294-302, 2013

[24] C. -H. Yu, Application of Maple: double improper integrals as examples, Proceedings of 2013 Information Education and Technology Application Conference, Taiwan, pp. 1-5, 2013.

[25] C. - H. Yu, Application of Maple on the evaluation of four types of double integrals, Proceedings of 2013 Business Innovation and Development Conference, Taiwan, B20130117002, 2013.

[26] L. Flatto, Advanced Calculus, Baltimore: The Williams \& Wilkins, p405, 1976.

[27] T. M. Apostol, Mathematical Analysis, 2nd ed., Boston: Addison-Wesley, 1975. 